\title{
ARTICLE
}

\section{Thermal neutron distribution in the beam line tunnel of the KEK electron/positron injector linac}

\author{
Takahiro Oyama $^{\mathrm{a}^{*}}$, Hiroshi Iwase ${ }^{\mathrm{a}}$, Akihiro Toyoda ${ }^{\mathrm{a}}$, Naoto Yoshihara ${ }^{\mathrm{b}}$ and Toshiya Sanami ${ }^{\mathrm{a}}$ \\ ${ }^{a}$ High Energy Accelerator Research Organization, Oho, Tsukuba-shi, Ibaraki-ken, 305-0801, Japan; ${ }^{b}$ Tokyo Nuclear Services Co., \\ Ltd., Midorigahara, Tsukuba-shi, Ibaraki-ken, 300-2646, Japan
}

\begin{abstract}
The thermal neutron flux distribution formed in the KEK electron/positron injector linac (KEK-linac) tunnel by the positron production beam operation was measured using a gold foil activation technique. The measured data were compared with the calculation results of the PHITS code. The data indicate that the thermal neutrons were locally distributed within a range of $15-20 \mathrm{~m}$ of the target position. The thermal neutron flux obtained using the PHITS code reproduced the experimental data at the target location. We obtained coefficient " $C$ " for KEK-linac tunnel from experimental result and calculation as 2.25 that is used in empirical formula to obtain thermal neutron flux, $\phi_{\mathrm{h}}=C Q / S$, where $Q$ is neutron source intensity, $S$ is the area of tunnel surface.
\end{abstract}

Keywords: air activation; neutron yield; thermal neutron distribution; gold foil activation; Monte Carlo

\section{Introduction}

The KEK-linac is a multipurpose 600-m linear accelerator for supplying electrons and positrons beams to two rings of the SuperKEKB collider and two light source rings PF/PF-AR simultaneously. During positron production by the $3.3 \mathrm{GeV}$ electron beam, a $2.2-\mathrm{kW}$ beam loss occurs regularly at the tungsten target, which has a thickness of 4 radiation-lengths.

Air activation induced by beam operation is an important concern in radiation safety for high-energy accelerator facilities. While the activation level of electron accelerators is generally low in comparison to hadron facilities, the KEK-linac will be operated with $\mathrm{kW}$ levels of beam loss continuously for the upcoming SuperKEKB project; thus, accurate evaluation of air activation is important for the radiation shielding strategy.

In this study, we focused on radioactive ${ }^{41} \mathrm{Ar}$ production, as it has a large production cross sections of 0.66 barn [1], a relatively long half-life of 1.8 hours. It makes a considerable contribution to the total amount of air activation.

Analytical estimation of the thermal neutron flux can be obtained from two simple empirical equations. First, Mao's equation gives the number of neutrons per electron loss as follows:

$$
Y_{n}=8 \times 10^{-6} E_{0}\left(\mathrm{Z}^{0.5}+0.12 \mathrm{Z}^{1.5}-0.001 \mathrm{Z}^{2.5}\right)
$$

\footnotetext{
*Corresponding author. Email: takahiro.oyama@kek.jp
}

where $Y_{\mathrm{n}}$ is the number of neutrons from giant dipole resonance (GDR) reactions, $Z$ is the target material atomic number, and $E_{0}$ is the electron energy in $\mathrm{MeV}$ [2]. The thermal neutron flux can be obtained from the number of neutrons using Patterson's equation:

$$
\phi_{t h}=C \frac{Q}{S}
$$

where $\phi_{\mathrm{h}}\left(/ \mathrm{cm}^{2} / \mathrm{s}\right)$ is the average thermal neutron flux in the room, $S$ is the inner surface area $\left(\mathrm{cm}^{2}\right)$ of the room, $Q$ is the neutron source intensity (neutrons/s) and $C$ is a coefficient as a fitting parameter. Patterson and Thomas recommended a $C$ value of 1.25 [3].

Ishikawa et al. confirmed that $C$ depends on the source neutron energy from calculations using the ANISN-W code. They proposed an average $C$ value of 4 [4]. Lee et al. estimated thermal neutron flux in a concrete room with simple shapes using a Monte Carlo code (MCNPX) [5]. As a result, their $C$ value was smaller than the value proposed by Ishikawa et al., 4, but larger than the values recommended by Patterson et al., 1.25 .

In addition to the source neutron energy dependence, $C$ is possibly affected by the room shape, size and absorption of neutrons by various surrounding materials such as the accelerator chamber, magnet, and other peripheral equipment [4]. Owing to these variables, it was still unclear whether $C$ values from previous studies are applicable to large accelerator facilities, specifically to the KEK-linac.

In this study, in order to obtain the most suitable $C$ value for the KEK-linac, the thermal neutron distribution 
caused by the electron beam loss during the positron production was measured and calculated.

\section{Materials and methods}

\subsection{Experimental setup}

Figure 1 shows a schematic diagram of the positron target unit. The primary electrons hit a tungsten crystal $14 \mathrm{~mm}$ thick and $4 \mathrm{~mm}$ in diameter. Positrons are created by pair production and then focused by a strong magnetic field produced by a flux concentrator (FC) before acceleration.

The KEK-linac is suitable for the measurement and Monte Carlo simulation of the thermal neutron distribution as its beam line tunnel has a simple linear shape (600 $\mathrm{m}$ long, $4 \mathrm{~m}$ wide and $5 \mathrm{~m}$ high). No heavy shielding is installed around the target.

The thermal neutron flux distribution was measured using gold foil activation technique with the $\mathrm{Cd}$ difference method. The bare and Cd-covered gold foils

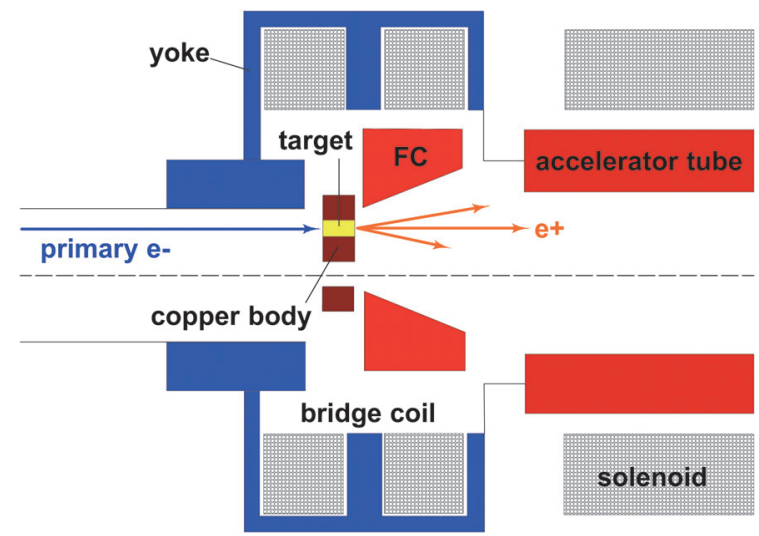

Figure 1. Schematic view of the positron production target unit (not to scale).

were packed in a polyethylene bags and placed along the KEK-linac tunnel. Figure 2 shows the plan and sectional views of the installation positions. The disk foils were $99.99 \%$ purity gold $\left({ }^{197} \mathrm{Au}\right)$ with a thickness of $20 \mu \mathrm{m}$ and a diameter of $6.0 \mathrm{~mm}$. Cd covers of 0.5 $\mathrm{mm}$ thick were used to sandwich the gold foil.

After setting the gold foils, the beam operation for positron production was performed. The $3.3 \mathrm{GeV}$ electron was incident on the tungsten target. The net beam operation duration was $2.35 \mathrm{~h}$, and the average beam current was $317.4 \mathrm{nA}$.
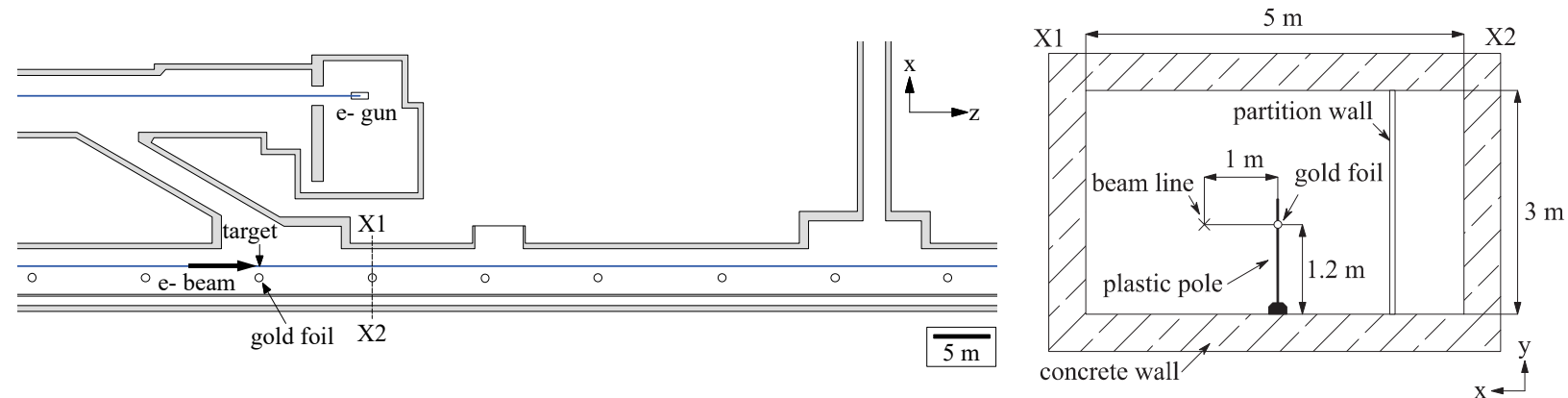

Figure 2. Experimental setup for the thermal neutron measurements at the KEK-linac. 

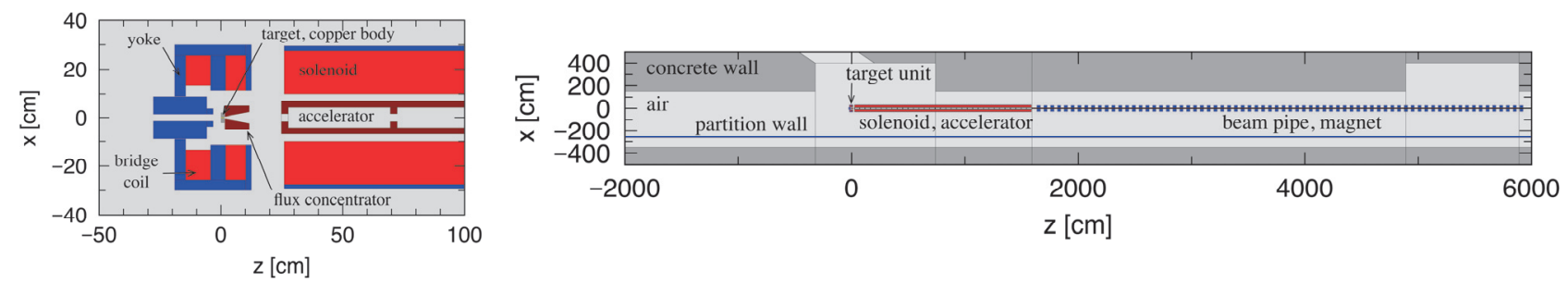

Figure 4. Top cross-section view of the PHITS calculation model of KEK-linac tunnel. (left) Closeup of the positron production target unit; (right) Beam line tunnel and the devices considered on the downstream side of the target.

where:

$A_{\text {bare }}=$ saturation activity of bare gold foil $(\mathrm{Bq} / \mathrm{g})$,

$A_{C d}=$ saturation activity of Cd-covered gold foil

$(\mathrm{Bq} / \mathrm{g})$,

$N_{A u}=$ total number of target nuclei in the gold foil,

$\sigma=98.65 \pm 0.09$ barn, the activation cross-section

by $2,200 \mathrm{~m} / \mathrm{s}$ neutrons [1],

$F_{t h}=1.038$, a correction factor for self-shielding in the gold foil [7],

$F_{C d}=1.013$, a correction factor for epithermal neutron absorbed into Cd [7],

$T=$ neutron temperature, and

$T_{0}=$ medium temperature.

Suppose that the energy spectrum of thermal neutrons in KEK-linac tunnel is Maxwellian, the term $\phi_{\mathrm{h}}$ can be defined as the integral over all thermal energy region below the Cd cutoff energy (about $0.5 \mathrm{eV}$ ).

\subsection{PHITS Monte Carlo model}

To investigate the process of the neutron production by electron beam loss at the tungsten target, attenuation of produced neutrons and thermalizaition in the KEK-linac tunnel, the neutron yields and the thermal neutron flux when $3.3 \mathrm{GeV}$ electron on the tungsten target were calculated using the PHITS 2.91 code [8].

Figure 4 shows the geometry for the PHITS calculation. The geometry description includes the KEK-linac tunnel structure; positron target unit; and beamline equipment at the downstream side of the target, such as accelerator tube, solenoids and magnets. The target position was defined as the origin of the coordinate $\operatorname{system}(\mathrm{x}=\mathrm{y}=\mathrm{z}=0 \mathrm{~m})$.

The electron beam energy incident on the target was

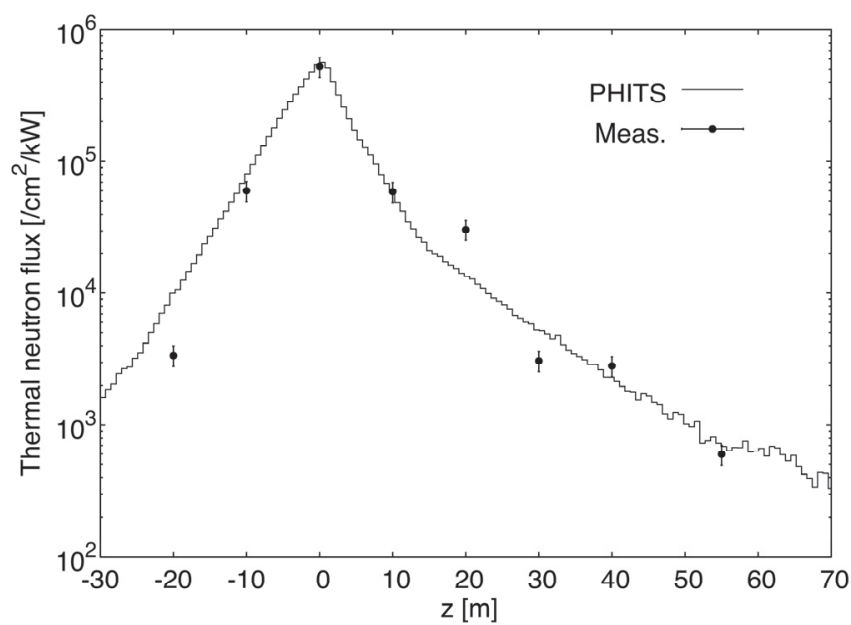

Figure 5. Comparison between experimental results using gold foils and the PHITS calculation.
3.3 GeV. The transport of electrons, positrons and photons was calculated by the EGS5 algorithm incorporated within the PHITS [9]. The neutron interaction was calculated by INCL and GEM models for high energy neutrons and by the JENDL-4.0 nuclear data library below $20 \mathrm{MeV}$.

The PHITS calculation outputs were the number of neutrons $Y_{\mathrm{n}}$ from GDR reactions, and the spatial distribution of the thermal neutron flux $\phi_{\text {th }}$ (up to $0.5 \mathrm{eV}$ ) in the KEK-linac tunnel. The number of GDR neutrons $Y_{\mathrm{n}}$ was obtained by scoring the neutrons up to $10 \mathrm{MeV}$ from photo nuclear interaction immediately after generation.

\section{Results and discussion}

\subsection{Thermal neutron distribution}

Figure 5 shows a comparison of the experimental data and PHITS calculations for the thermal neutron distribution along the KEK-linac tunnel. More than $90 \%$ of the thermal neutrons were locally distributed within a range of $15 \mathrm{~m}$ of the target position. Because of several small beam losses that occurred at the downstream side of the target, the thermal neutron distribution is asymmetrical with respect to the target position.

The thermal neutron flux obtained by PHITS reproduced the experimental data well. These results indicated that PHITS is properly described neutron production at the target and peripheral equipment, and, neutron thermalization.

Figure 6 shows the calculated thermal neutron distribution in a Y1-Y2 plane perpendicular to the beam axis; the distribution is indicated by a dashed line in

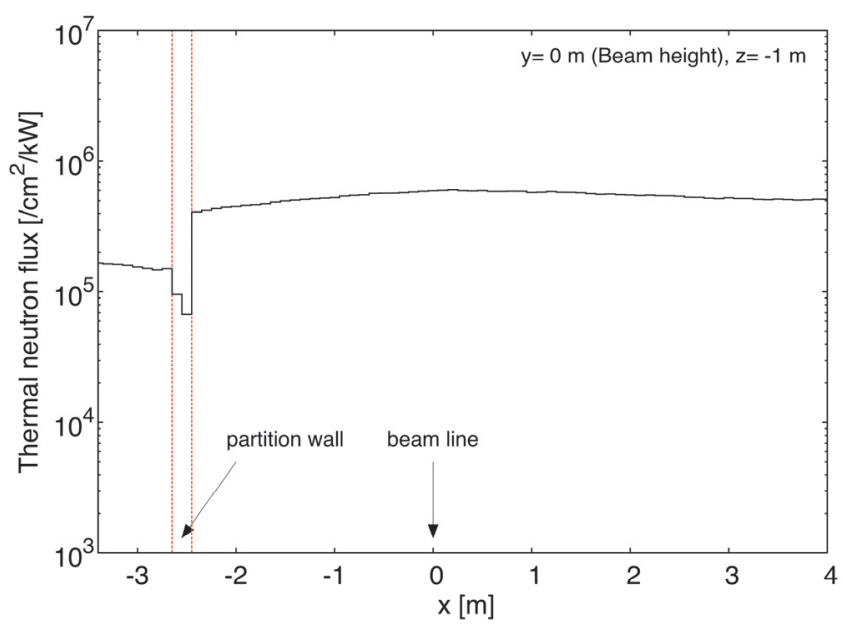

Figure 6. Calculated horizontal distribution of thermal neutrons. 
Figure 4. The thermal neutrons were uniformly distributed over the horizontal direction, except at the partition wall between the beamline area and a side room. Thus, the average thermal neutron flux in the KEK-linac can be obtained from the distribution in a beam direction.

\subsection{Derivation of experimental $C$ values}

In this section, we deduced the $C$ values for the KEK-linac tunnel based on the experimental results. During positron production, more than $90 \%$ of the thermal neutrons were distributed within $15 \mathrm{~m}$ of the target position. In this area, the average thermal neutron flux $\phi_{\mathrm{th}}$ and surface area $S$ of the concrete wall were estimated to be $1.42 \times 10^{5} / \mathrm{cm}^{2} / \mathrm{kW}$ and $5.46 \times 10^{6} \mathrm{~cm}^{2}$, respectively.

The neutron source intensity $Q$ was calculated using the PHITS code. According to the concept in Eq.1, $Q$ is the number of GDR neutrons induced by the electron beam loss, and it is equal to $Y_{\mathrm{n}}$. Figure 7 shows the energy spectrum of GDR neutrons generated at beam line device depicted in Figure 4. Then, by integrating the energy spectrum up to $10 \mathrm{MeV}, Y_{\mathrm{n}}$ was obtained from the GDR reaction. The PHITS calculation estimated $Y_{\mathrm{n}}$ to be $1.38 \times 10^{12}$ neutrons $/ \mathrm{kW}$. However, this $Y_{\mathrm{n}}$ value did not account for shielding effects such as neutron absorption. In order to take account for shielding effects

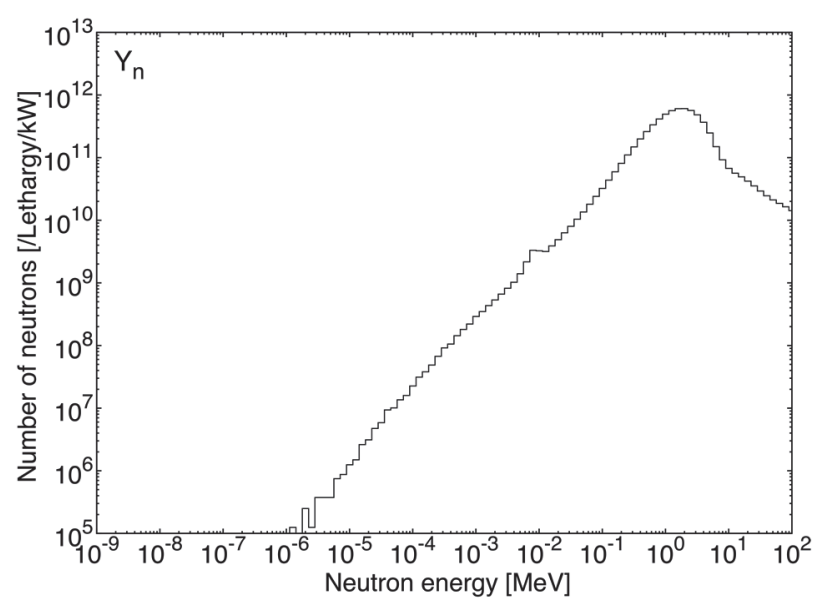

Figure 7. Calculated neutron energy spectra when neutrons were generated by photonuclear reaction.

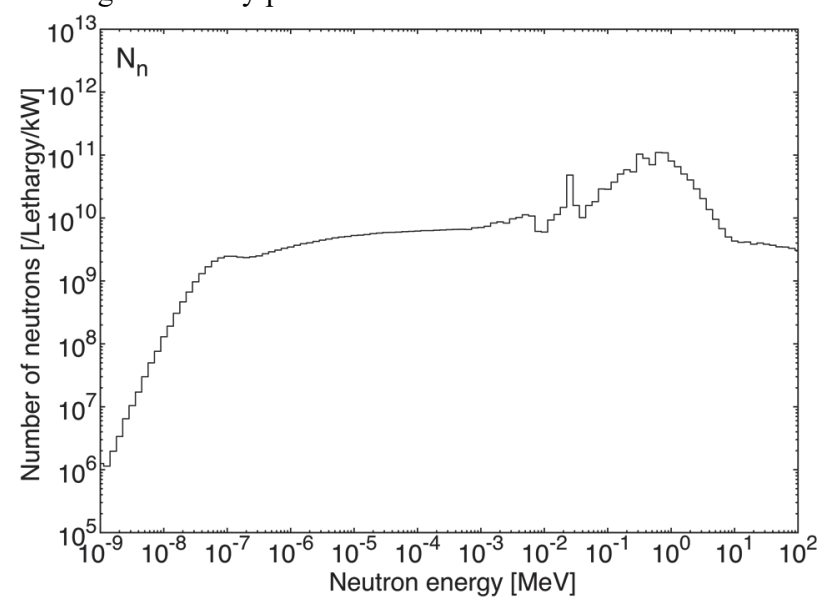

Figure 8. Calculated neutron energy spectra when generated photoneutrons reached the concrete wall. properly, the value $N_{\mathrm{n}}$ is defined as a quantity of the number of neutrons entering the concrete wall. The tally region of $N_{\mathrm{n}}$ was defined over entire the surface of the concrete wall in Figure 4. Figure 8 shows the energy spectrum of the neutrons. $N_{\mathrm{n}}$ was obtained from the energy spectrum to be $3.45 \times 10^{11}$ neutrons $/ \mathrm{kW}$, which is approximately 4 times lower than $Y_{\mathrm{n}}$. This indicates that the shielding effect of the beamline equipment is not negligible. Taking the neutron source intensity $Q$ as $N_{\mathrm{n}}$, the coefficient $C$ for the KEK-linac was estimated to be 2.25. In comparison with the $C$ values reported in previous studies, our result is smaller than Ishikawa's value (4) and larger than Patterson's value (1.25). Lee's value for $3 \mathrm{MeV}$ mono-energy neutrons, 2.43 , is close to the value we obtained.

\section{Conclusion}

To estimate the ${ }^{41} \mathrm{Ar}$ production in air, the thermal neutron flux distribution in the KEK-linac tunnel was measured by the gold foil activation method for $3.3 \mathrm{GeV}$ electrons on a tungsten target. The experimental results indicated that the thermal neutrons were locally distributed within $15 \mathrm{~m}$ of the target. The thermal neutron flux obtained by the PHITS code reproduced the experimental data at the target location. The coefficient $C$ value was determined to be 2.25 , using values for $\phi_{\mathrm{h}}$ and $N_{\mathrm{n}}$ obtained from experimental data and the PHITS code.

The methodology of this study will be applied to the experiments with another accelerator facility, including the particle accelerator, to develop a more practical technique for the estimation of $\phi_{\mathrm{th}}$ in the beamline tunnel that replaces Patterson's equation, $\phi_{\mathrm{h}}=C Q / S$.

\section{References}

[1] S.F. Mughabghab, et al., Neutron Cross Sections Vol. 1, Academic Press, New York (1981).

[2] X. Mao, et al., Giant dipole resonance neutron yields produced by electrons as a function of target material and thickness, Health Phys., 70 (1996), pp. 207.

[3] H. W. Patterson, et al., Accelerator Health Physics, Academic Press, New York (1973).

[4] T. Ishikawa, et al., Thermalization of accelerator-produced neutrons in a concrete room, Health Phys., 60 (2) (1991), pp.209.

[5] C.W. Lee, et al., Analysis of the neutron thermalization in an accelerator room, J. Nucl. Sci. Technol., Supplement 5 (2008), pp. 42-45.

[6] F. L. Bronson, Validation of the accuracy of the LabSOCS software for mathematical efficiency calibration of Ge detectors for typical laboratory samples, J. Radioanal. Nucl. Chem., 255 (2003), pp. 137-141.

[7] K.H. Beckurts, et al., Neutron Physics, SpringerVerlag (1964).

[8] T. Sato, et al., Particle and heavy ion transport code system PHITS, Version 2.52, J. Nucl. Sci. Technol., 50 (9) (2013), pp. 913-923.

[9] H. Hirayama, et al., SLAC-R-730 (2005). 